\title{
Eight new MSPs in NGC 6440 and NGC 6441
}

\author{
Paulo C. C. Freire*, Scott M. Ransom ${ }^{\dagger}$, Steve Bégin**, Ingrid H. Stairs**, Jason W. \\ T. Hessels ${ }^{\ddagger}$, Lucille H. Frey ${ }^{\S}$ and Fernando Camilo ${ }^{\mathbb{I}}$ \\ ${ }^{*}$ N.A.I.C., Arecibo Observatory, HC3 Box 53995, PR 00612, U.S.A.; pfreire@ naic .edu \\ ${ }^{\dagger}$ N.R.A.O., 520 Edgemont Road, Charlottesville, VA 22903, U.S.A.; sransomenrao. edu \\ ${ }^{* *}$ Department of Physics and Astronomy, University of British Columbia, Vancouver, BC V6T 1Z1, Canada \\ "Astronomical Institute “Anton Pannekoek", University of Amsterdam, 1098 SJ Amsterdam, The Netherlands \\ ${ }^{\S}$ Department of Astronomy, Case Western Reserve University, Cleveland, OH 44106 \\ ${ }^{\mathbb{I}}$ Columbia Astrophysics Laboratory, Columbia University, New York, NY 10027
}

\begin{abstract}
We report the discovery of five new millisecond pulsars in the globular cluster NGC 6440 and three new ones in NGC 6441; each cluster has one previously known pulsar. Four of the new pulsars are found in binary systems. One of the new pulsars, PSR J1748-2021B in NGC 6440, is notable for its eccentric $(e=0.57)$ and wide $\left(P_{b}=20.5\right.$ days $)$ orbit. If the rate of advance of periastron is due solely to general relativity, we can estimate of the total mass of this binary system: $2.92 \pm 0.20 M_{\odot}$. This would imply an anomalously large mass for this pulsar, which could introduce important constraints in the study of the equation of state for cold neutron matter.
\end{abstract}

Keywords: Millisecond Pulsars; Binary Pulsars; Precision Timing

PACS: $97.60 . \mathrm{Gb} ; 97.60 . J d ; 97.60 .-\mathrm{s} ; 97.80 .-\mathrm{d}$;

\section{DISCOVERY}

The recent discovery of 30 millisecond pulsars (MSPs) in Terzan 5 [1, 2], made using the GBT's S-band receiver and the pulsar spigot [3] shows that this observing system (henceforth GBT/S/PS) has an unprecedented sensitivity to MSPs outside the Arecibo sky. This is only partly due to the factor of 3 higher gain of the GBT as compared to the Parkes telescope. The higher observing frequency $(\sim 2 \mathrm{GHz})$, large observing bandwidth $(600 \mathrm{MHz})$, and relatively fine frequency resolution $(\sim 0.78 \mathrm{MHz})$ provide enough frequency and time resolution to detect faint MSPs at high dispersion measures (DM). An impressive demonstration of this is the discovery of PSR J1748-2446ad, the fastest spinning pulsar known $(P=1.396 \mathrm{~ms})$. This was found in Terzan 5, where the average DM of the pulsars is $239 \mathrm{~cm}^{-3} \mathrm{pc}$. This motivated us to search for MSPs in other promising GCs with this system.

For the first phase of the survey, we selected the globular clusters (GCs) NGC 6388, NGC 6440 and NGC 6441 based on their high stellar interaction rates at the core, $\Gamma_{c}$ [4]. Despite the large $\Gamma_{c}$ s of these GCs, only two pulsars were known in them: PSR B1745-20A (NGC6440A, [5]) and PSR J1750-37A (NGC 6441A, [6]). The spin periods and DMs of NGC 6440A and NGC 6441A are $288.6 \mathrm{~ms}$ and $111.6 \mathrm{~ms}$ and 220 and $234 \mathrm{~cm}^{-3} \mathrm{pc}$, respectively. We assumed that the lack of other known pulsars in these high-DM clusters was largely due to a bias of the earlier surveys against the detection of MSPs. The GBT/S/PS system can greatly reduce this bias.
With full track observations made in 2005, we discovered eight new MSPs, five of them in NGC 6440, three in NGC 6441 and none in NGC 6388. Preliminary results on these objects were presented in Bégin (2006)[7]. Their pulse profiles, together with those of the previously known objects, are presented in Figure 10 These pulse profiles were obtained by adding all detections of the pulsars, carefully excluding all sub-integrations that are corrupted by RFI.

\section{LUMINOSITY DISTRIBUTION}

In Figure 2, we make cumulative plots of the pseudo luminosities of the pulsars in NGC 6440, NGC 6441 and Terzan 5, the latter based on the flux density estimates reported in [1]. This allows a direct comparison of the sizes of the pulsar populations of these clusters. The observing system and observing frequency are the same and the DMs of the pulsars are very similar, making the search similarly sensitive to fast-spinning pulsars. The software used to do the searches is the same, and the software used to make the flux density measurements is similar (but not exactly the same). All these factors make the flux densities directly comparable; the pseudo-luminosities will then be directly comparable as well if the cluster distances are accurate. We have used the latest distance estimates for these GCs (5.5 kpc for Terzan 5, [8], $8.2 \mathrm{kpc}$ for NGC 6440 and $13.5 \mathrm{kpc}$ for NGC 6441 [9]) to estimate the pulsar pseudo-luminosities, but caution that these distances still have large systematic uncertainties. 


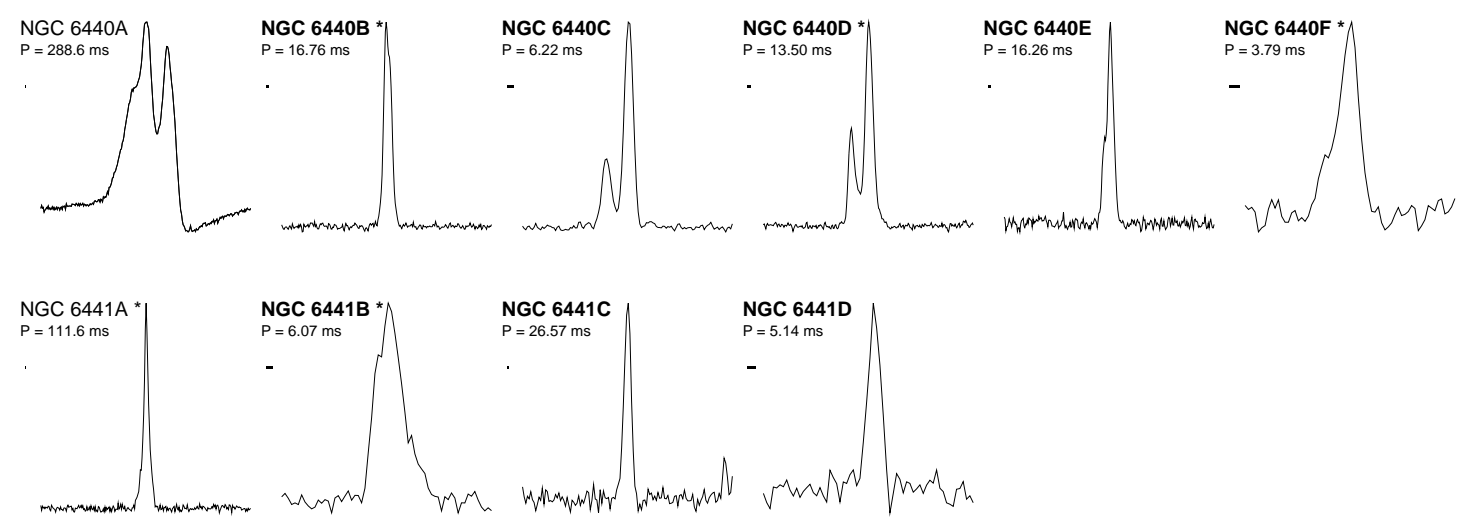

FIGURE 1. Average $1950 \mathrm{MHz}$ pulse profiles for the 10 pulsars known in the GCs NGC 6440 and NGC 6441. These profiles cover one full rotation. The pulsars in binary systems have an asterisk after their names, and the newly discovered pulsars have their names in boldface. The horizontal width of the rectangles indicates the system's total time resolution, including the effects of dispersive smearing, relative to each pulsar's spin period. The dip in power after the main pulse of NGC 6440A might be a SPIGOT artifact.

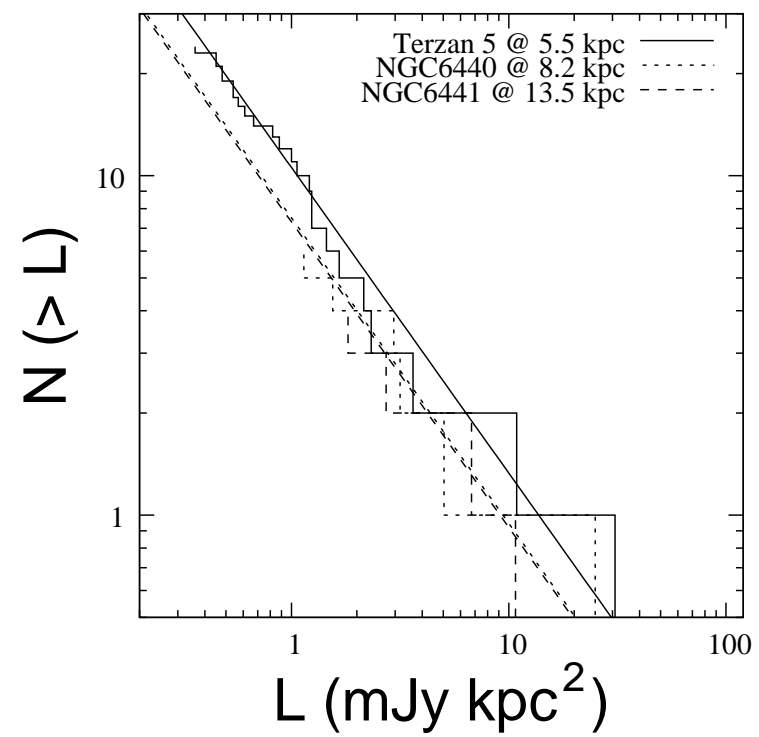

FIGURE 2. Cumulative distribution of pseudo luminosities at $1950 \mathrm{MHz}$ for the pulsars in NGC 6440, NGC 6441 and Terzan 5, assuming the most recent distance estimates.

We have fitted a luminosity function of the type $N(>L)=k_{1} L^{-0.9}$ [10] to the observed pulsar pseudoluminosity distribution of each cluster, where $N(>L)$ is the number of pulsars above a pseudo luminosity of $L$ and $k_{1}$ is the number of pulsars brighter than $1 \mathrm{mJy} \mathrm{kpc}^{2}$. We obtain $k_{1}=10.6,7.5$ and 7.3 for Terzan 5, NGC 6440 and NGC 6441 respectively. The clusters seem to have similarly sized pulsar populations, as expected from their stellar interaction rates: Terzan 5, NGC 6440 and NGC $6441, \Gamma_{c}$ have $6.3,6.3$ and $8.6 \%$ of the total stellar interaction rate of all Galactic GCs. We detect more pulsars in Terzan 5 because that cluster is closer to us.

\section{A SUPER-MASSIVE NEUTRON STAR?}

One of the most interesting new discoveries is PSR J1748-2021B. This is a 16.7-ms pulsar in a wide $\left(P_{b}=20.55\right.$ days $)$ and eccentric $(e=0.57)$ orbit with $\mathrm{a} \sim 0.1 M_{\odot}$ companion. This orbital eccentricity has allowed a measurement of the rate of advance of periastron: $\dot{\omega}=0.00391(18)^{\circ} \mathrm{yr}^{-1}$.

Assuming that $\dot{\omega}$ is fully relativistic (as argued in [4]), that implies a total system mass of $2.92 \pm 0.20 M_{\odot}$. In Figure 3] we present the mass constraints on the components graphically, with probability distribution functions for the mass of the pulsar and the companion presented above and on the right respectively [4].

The total mass of this system suggests that it is a double neutron star (DNS) binary. However, the probability that it lies within the range of neutron star masses that have been precisely measured to date, indicated in Fig. 3 by grey bars, is only $0.10 \%$. This eventuality would require improbably low orbital inclinations, between $\sim 4^{\circ}$ and $\sim 5^{\circ}$.

The median pulsar mass is $2.74 M_{\odot}$, with the lower and upper $1 \sigma$ limits at 2.52 and $2.95 M_{\odot}$. The pulsar has only a $1.0 \%$ probability of being less massive than $2.01 M_{\odot}$, and a $1.0 \%$ probability of being more massive than $3.24 M_{\odot}$. If this high mass value is confirmed, it would be the largest pulsar mass measured, surpassing that for PSR B1516+02B in the GC M5 $\left(1.96_{-0.12}^{+0.09} M_{\odot},[13]\right)$. Confirmation of such a large neutron star mass would have profound consequences for the study of dense matter, since it excludes almost all known equations of state [14]. Since DNS systems have similar masses, this might even imply that the end products of the coalescence of DNS systems might themselves be stable as super-massive neutron stars! 


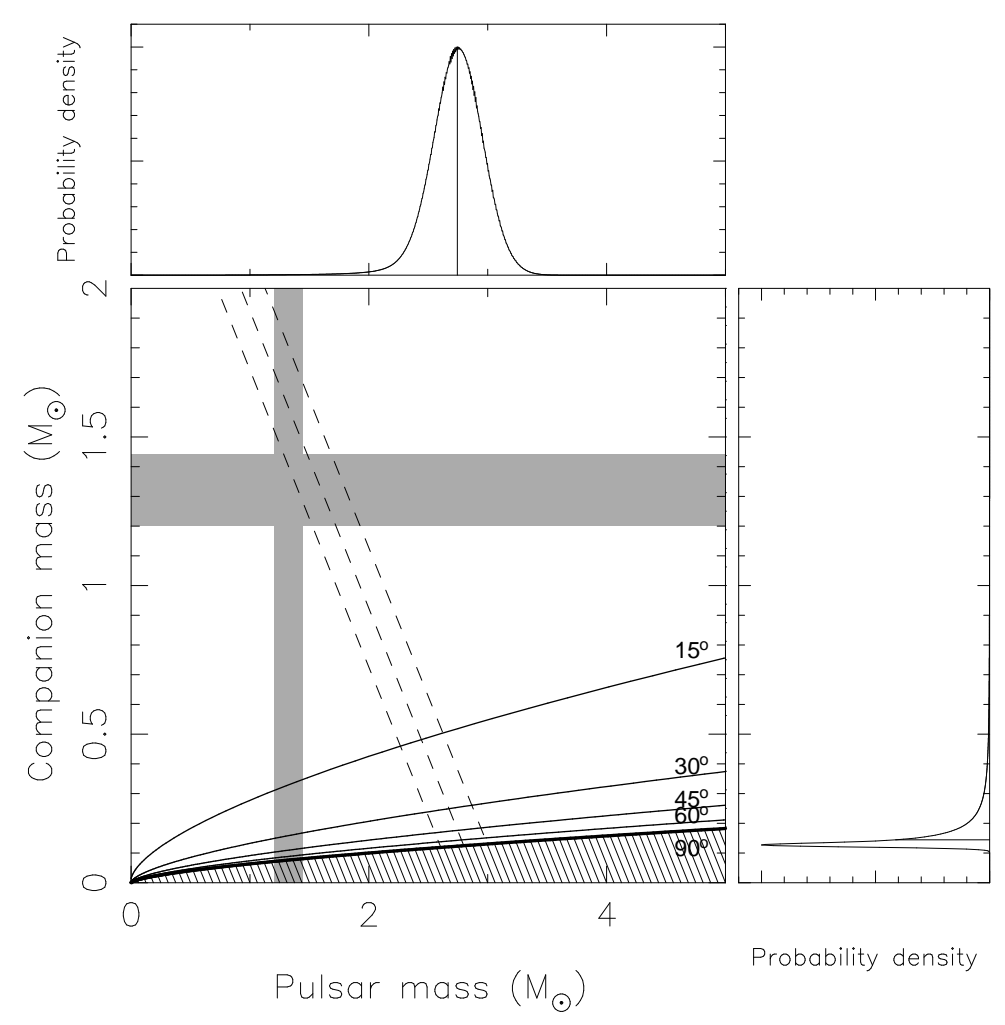

FIGURE 3. Constraints on the masses of NGC 6440B and its companion. The hatched region is excluded by knowledge of the mass function and by $\sin i \leq 1$. The diagonal dashed lines correspond to a total system mass that causes a general-relativistic $\dot{\omega}$ equal or within $1-\sigma$ of the measured value. The five solid curves indicate constant orbital inclinations. The gray bars indicate the range of precisely measured neutron star masses, this goes from $\sim 1.20 M_{\odot}$ for the companion of PSR J1756-2251[11] to 1.44 $M_{\odot}$ for PSR B1913+16 [12]. We also display the probability density function for the mass of the pulsar (top) and the mass of the companion (right), and mark the respective medians with vertical (horizontal) lines.

\section{ACKNOWLEDGMENTS}

The National Radio Astronomy Observatory is a facility of the National Science Foundation operated under cooperative agreement by Associated Universities, Incorporated.

\section{REFERENCES}

1. S. M. Ransom, J. W. T. Hessels, I. H. Stairs, P. C. C. Freire, F. Camilo, V. M. Kaspi, and D. L. Kaplan, Science 307, 892-896 (2005).

2. J. W. T. Hessels, S. M. Ransom, I. H. Stairs, P. C. C. Freire, V. M. Kaspi, and F. Camilo, Science 311, 1901-1904 (2006).

3. D. L. Kaplan, R. P. Escoffier, R. J. Lacasse, K. O’Neil, J. M. Ford, S. M. Ransom, S. B. Anderson, J. M. Cordes, T. J. W. Lazio, and S. R. Kulkarni, P.A.S.P. 117, 643-653 (2005).

4. P. C. C. Freire, S. M. Ransom, S. Bégin, I. H. Stairs, J. W. T. Hessels, L. H. Frey, and F. Camilo, ArXiv e-prints 0711.0925 (2007).
5. A. G. Lyne, R. N. Manchester, and N. D'Amico, Ap.J. 460, L41-L44 (1996).

6. A. Possenti, A. Corongiu, D. Manchester, F. Camilo, A. Lyne, N. D'Amico, J. Sarkissian, F. Ferraro, and G. Cocozza, Chinese Journal of Astronomy and Astrophysics Supplement 6, 176-180 (2006).

7. S. Bégin, A Search for Fast Pulsars in Globular Clusters, Master's thesis, University of British Columbia (2006).

8. S. Ortolani, B. Barbuy, E. Bica, M. Zoccali, and A. Renzini, ArXiv e-prints 0705.4030 (2007).

9. E. Valenti, F. R. Ferraro, and L. Origlia, A.J. 133, 1287-1301 (2007).

10. J. W. T. Hessels, S. M. Ransom, I. H. Stairs, V. M. Kaspi, and P. C. C. Freire, Ap.J., 670, 363 (2007).

11. A. J. Faulkner, M. Kramer, A. G. Lyne, R. N. Manchester, M. A. McLaughlin, I. H. Stairs, G. Hobbs, A. Possenti, D. R. Lorimer, N. D'Amico, F. Camilo, and M. Burgay Ap.J.Lett., 618, L119-L122 (2005).

12. J. M. Weisberg, and J. H. Taylor, "The Relativistic Binary Pulsar B1913+16," in Radio Pulsars, edited by M. Bailes, D. J. Nice, and S. Thorsett, Astronomical Society of the Pacific, San Francisco, 2003, pp. 93-98.

13. P. C. C. Freire, A. Wolszczan, M. van den Berg, and J. W. T. Hessels, Ap.J., submitted (2007).

14. J. M. Lattimer, and M. Prakash, Phys. Rep. 442, 109-165 (2007). 